Journal for ImmunoTherapy of Cancer

\section{DNA damage repair pathway alterations in metastatic clear cell renal cell carcinoma and implications on systemic therapy}

To cite: Ged Y, Chaim JL, DiNatale RG, et al. DNA damage repair pathway alterations in metastatic clear cell renal cell carcinoma and implications on systemic therapy. Journal for ImmunoTherapy of Cancer 2020;8:e000230. doi:10.1136/ jitc-2019-000230

- Additional material is published online only. To view please visit the journal online (http://dx.doi.org/10.1136/jitc2019-000230).

Accepted 25 March 2020

\section{ABSTRACT}

Background Loss-of-function alterations in DNA damage repair (DDR) genes are associated with human tumorigenesis and may determine benefit from immune-oncology (1/0) agents as shown in colon cancer. However, biologic significance and relevance to l/0 in metastatic clear cell RCC (cCRCC) are unknown.

Methods Genomic data and treatment outcomes were retrospectively collected for patients with metastatic ccRCC. Tumor and germline DNA were subject to targeted next generation sequencing across $>400$ genes of interest, including 34 DDR genes. Patients were dichotomized according to underlying DDR gene alteration into (1) deleterious DDR gene alterations present (Del DDR); (2) wildtype (WT) and variants of unknown significance (VUS) DDR gene alterations present (WT/NUS DDR). Association between DDR status and therapeutic benefit was investigated separately for I/0 and vascular endothelial growth factor (VEGF) tyrosine kinase inhibitor (TKI) therapy.

Results Del DDR were detected in 43/229 patients (19\%). The most frequently altered genes were CHEK2 and ATM. Clonality analysis was performed in 27 somatic DDR mutations and 17 were clonal (63\%). For patients with $\mathrm{l} / 0$ treatment, Del DDR status was associated with superior overall survival (log-rank $p=0.049$ ); after adjusting for International Metastatic Renal Cell Carcinoma Database Consortium risks and extent of prior therapy, the HR for Del DDR was 0.41 (95\% Cl: 0.14-1.14; $p=0.09$ ). No association was seen with VEGF-TKI treatment (log-rank $p=0.903$ ). Conclusion Del DDR alterations are recurrent genomic events in patients with advanced RCC and were mostly clonal in this cohort. Loss-of-function events in these genes may affect outcome with $\mathrm{I} / 0$ therapy in metastatic RCC, and these hypothesis-generating results deserve further study.

(c) Author(s) (or their employer(s)) 2020. Re-use permitted under CC BY-NC. No commercial re-use. See rights and permissions. Published by BMJ.

For numbered affiliations see end of article.

Correspondence to

Dr Martin H Voss;

vossm@mskcc.org tyrosine kinase inhibitors (TKIs) were the most effective treatment options both in the firstline $^{23}$ and refractory line settings. ${ }^{45}$ Recently, immune-oncology (I/O) agents including monoclonal antibodies targeting the programmed cell death-1/programmed cell death-ligand 1 (PD-1/PD-L1) and cytotoxic t-cell lymphocyte-4 (CTLA-4) checkpoints 67 have revolutionized the treatment landscape for patients with metastatic ccRCC representing a new standard of care and a backbone in evolving therapeutic strategies. However, not all patients benefit equally from $\mathrm{I} / \mathrm{O}$ agents, and thus far, no validated biomarkers are currently used to guide treatment selection for individual patients.

The DNA damage repair (DDR) pathways consist of multiple interconnected cellular signaling pathways that are activated in response to DNA damage. As a network, these pathways can coordinate a cascade of events, including cell cycle arrest, regulation of DNA replication, and the repair or bypass of DNA damage. ${ }^{8}$ Since multiple key DDR genes have functional overlap, defects in DDR genes can be a source of genomic instability and may sensitize responses to $\mathrm{I} / \mathrm{O}$ agents possibly through the production of tumor-associated neoantigen and/or the activation of the stimulator of interferon genes (STING) pathway. ${ }^{9} 10$ This conceptually has been clinically supported by the robust clinical benefit of solid tumors exhibiting high microsatellite instability (MSI-H) and subsequent high hypermutation rates to the anti-PD-1 antibody pembrolizumab. ${ }^{11}$ More recently, the presence of deleterious DDR gene alterations in bladder cancer was shown to be associated with improved clinical outcomes in patients treated with $\mathrm{I} / \mathrm{O}$ agents. ${ }^{12}$ 
Based on these observations, we retrospectively examined a cohort of 229 patients with metastatic ccRCC at the Memorial Sloan Kettering Cancer Center (MSKCC) who underwent targeted exon sequencing of tumor tissue by next generation sequencing (NGS) to define the frequency of DDR gene alterations. We then described the clinical phenotype of patients harboring deleterious DDR gene alterations in their tumors and studied their significance for clinical outcomes with standard systemic therapies (I/O agents and VEGF-TKI).

\section{METHODS}

\section{Study subjects}

Patients treated at MSKCC for metastatic RCC between January 2013 and October 2017 were retrospectively identified from an institutional database using an institutional review board approved protocol. Eligible patients were those with metastatic ccRCC who received anti-kidney cancer systemic therapy and underwent tumor-directed molecular profiling by targeted NGS using our institutional Memorial Sloan Kettering Integrated Mutation Profiling of Actionable Cancer Targets (MSK-IMPACT) platform. ${ }^{13}$ Patients' demographics, International Metastatic Renal Cell Carcinoma Database Consortium (IMDC) risk status, treatment details, therapeutic outcomes and survival status were recorded.

\section{Genomic sequencing analysis}

Tumors and matched normal blood samples from patients were sequenced using MSK-IMPACT, ${ }^{13}$ a targeted NGS platform performed in a Clinical Laboratory Improvement Amendments (CLIA) certified laboratory that consists of a targeted panel with $>400$ cancer-associated genes including 34 DDR genes. Raw sequencing data alignment was performed to human genome and mutations were called using our previously validated pipeline. ${ }^{13} 14$ Dedicated analysis for germline variants was conducted across 76 genes associated with cancer predisposition, including 21 DDR genes in a subset of patients who had provided consent for germline sequencing.

Copy number (CN) analysis was estimated by FACETS (Fraction and Allele-Specific Copy Number Estimates from Tumor Sequencing) V.0.5.6. ${ }^{15}$ A critical value (cval) of 100 was used to run the algorithm. To estimate the fraction of $\mathrm{CN}$-altered genome, we followed an approach similar to the one described by Endesfelder et al. ${ }^{16}$ Segments with absolute $\mathrm{CN}$ log-ratio $\geq 0.2$ were considered to be non-diploid. The length of the CN-altered segments was divided by the sum of all segments spanning across autosomes.

\section{DDR gene mutations' identification and determination of functionality}

In all patients included in the study, DDR gene mutations were identified from a panel of 34 DDR genes (eMethods, online supplementary material), which included genes in the checkpoint (CP), homologous recombination repair (HRR), mismatch repair (MMR), base excision repair
(BER), Fanconi anemia (FA) and nucleotide excision repair (NER) pathways. This gene panel was identified from prior work within our genitourinary group.

Patients were dichotomized into two categories (1) deleterious DDR: including patients harboring deleterious DDR alterations at the somatic and/or germline level; (2) wild type/VUS DDR: including patients with tumors determined to be DDR wild type and those whose tumors contained VUS DDR gene alterations. The following alterations were considered deleterious: truncating mutations (including frameshift insertion/ deletion, nonsense, or splice site alterations) and functionally validated missense mutations per the annotation of the functionality in Catalogue of Somatic Mutations in Cancer (COSMIC) database ${ }^{17}$ using the FATHMM-MKL (Functional Analysis Through Hidden Markov ModelsMultiple Kernel Learning) software (http://fathmm. biocompute.org.uk). ${ }^{18} \mathrm{CN}$ alterations were not included in the definition of deleterious DDR alterations. For the germline events, the pathogenicity of each DDR germline mutation was determined as per the American College of Medical Genetics (ACMG) criteria. ${ }^{19}$ DDR gene alterations that were not deleterious as per the above methods were categorized as VUS.

To determine the clonality of the DDR somatic mutations, we estimated the cancer cell fraction (CCF) as described by McGranahan et al. ${ }^{20}$ This method integrates a mutation's variant allelic frequency, tumor purity and base-specific ploidy and re-normalizes it to calculate the CCF estimates (range: $0-1$ ), with values closer to 1 representing a higher likelihood of clonality. In our analysis, we considered CCF values $\geq 0.75$ as likely clonal.

\section{Clinical outcome analysis and statistical endpoints}

Baseline characteristics were summarized as frequencies and proportions for categorical variables and medians and ranges for continuous variables. Categorical variables were compared using Fisher's exact test and continuous variables were compared using the Wilcoxon test. Studied clinical outcomes included overall survival (OS), progression-free survival (PFS) and best objective response rate (ORR). For OS and PFS, Kaplan-Meier curves were generated and compared by log-rank test. Cox proportional hazards regression for OS included as covariates deleterious DDR status, IMDC risk category at the start of therapy (favorable/intermediate/poor) for I/O and TKI analysis as a three-level categorical variable and number of prior lines of therapy received for $\mathrm{I} / \mathrm{O}$ analysis as a continuous variable. The adjusted HRs for DDR and their 95\% CIs are reported. Best ORR was compared between DDR groups using Fisher's exact test.

Separate investigations tested the association of DDR status and treatment outcomes on I/O and VEGF-TKI therapies. These analyses were run independently with no intended plan to formally compare outcomes per DDR status with one treatment modality versus the other. With that, patients could contribute to both analyses. For the $\mathrm{I} / \mathrm{O}$ analysis, we included patients who were previously 
$\mathrm{I} / \mathrm{O}$ naive and now first received I/O treatment including anti-PD-1 and anti-PD-L1 as a single agent or combined with anti-CTLA-4 antibodies. No combinational treatments of I/O with VEGF-TKI were included in this analysis. For the TKI analysis, we included patients previously unexposed to VEGF-directed TKI agents and investigated the association between DDR alterations and clinical outcomes with VEGF-TKI therapy.

In each analysis, OS was calculated for all patients from the date of starting the studied systemic therapy in each analysis including I/O and VEGF-TKI, respectively (first received $\mathrm{I} / \mathrm{O}$ in the $\mathrm{I} / \mathrm{O}$ analysis and first-line VEGF-TKI in the TKI analysis), until death, censoring those patients who were alive at the date of last follow-up. Analysis of PFS and ORR was restricted to patients with formal radiographic assessment per Response Evaluation Criteria in Solid Tumors (RECIST) V.1.1 ${ }^{21}$ performed by a dedicated research radiologist. PFS was assessed from the date the patient initiated the studied systemic therapy in each analysis to the date of progression by RECIST V.1.1 or death. Patients who did not progress were censored at the date of their last scan. Median follow-up was calculated in censored patients. Best ORR was determined as per response assessment by RECIST V.1.1.

All tests were evaluated for statistical significance at alpha level 0.05 . Statistical analysis was performed using SAS V.9.4.

\section{RESULTS}

\section{Patients' characteristics}

A total of 229 patients were included, all of whom had received systemic therapy for metastatic ccRCC at our center. Baseline characteristics are summarized in table 1. The majority of patients had undergone nephrectomy;
$37 \%$ had been diagnosed with de novo metastatic disease. IMDC risk at the start of first-line systemic therapy was favorable in $74(34 \%)$, intermediate in $113(53 \%)$, poor in $27(13 \%)$ and could not be determined in 15 patients. Two hundred forty-two tumors from 229 patients were profiled using MSK-IMPACT with germline comparison. Additionally, 171/229 (75\%) had consented to a protocol to explore hereditary predisposition and underwent dedicated NGS testing for germline events. NGS was predominantly performed in samples collected before the initiation of treatment (90\%; 206 patients of 229). Among the two treatment analyses, the proportions of primary versus metastatic biopsies were equally balanced (online supplementary table S1).

\section{NGS findings}

Across the 229 patients, 48 deleterious DDR gene alterations were identified in 43 patients (19\% of the entire cohort), including 35 (73\%) somatic and 13 (27\%) germline deleterious DDR gene alterations (online supplementary table S2). Five patients $(2 \%)$ had concurrent deleterious DDR gene alterations in two genes (three patients had both somatic and germline mutations, two patients had two somatic mutations).

The most frequently altered genes were CHEK $2(\mathrm{n}=10$; including three somatic and seven germline), $\operatorname{ATM}(\mathrm{n}=8$; all somatic), $M S H 6(\mathrm{n}=4$; including three somatic and one germline) and MUTYH ( $\mathrm{n}=4$; all germline). Alterations were noted across all pathways, including CP $(n=20)$, HRR $(n=9)$, MMR $(n=7)$, BER $(n=5)$, FA $(n=4)$, and NER $(n=3)$. Functionally, 33 out of the 48 alterations $(69 \%)$ were in genes involved in double-strand break repair and signaling processes (CP, HRR and FA pathways) (online supplementary table $\mathrm{S} 2$ ).

Table 1 Patients' characteristics

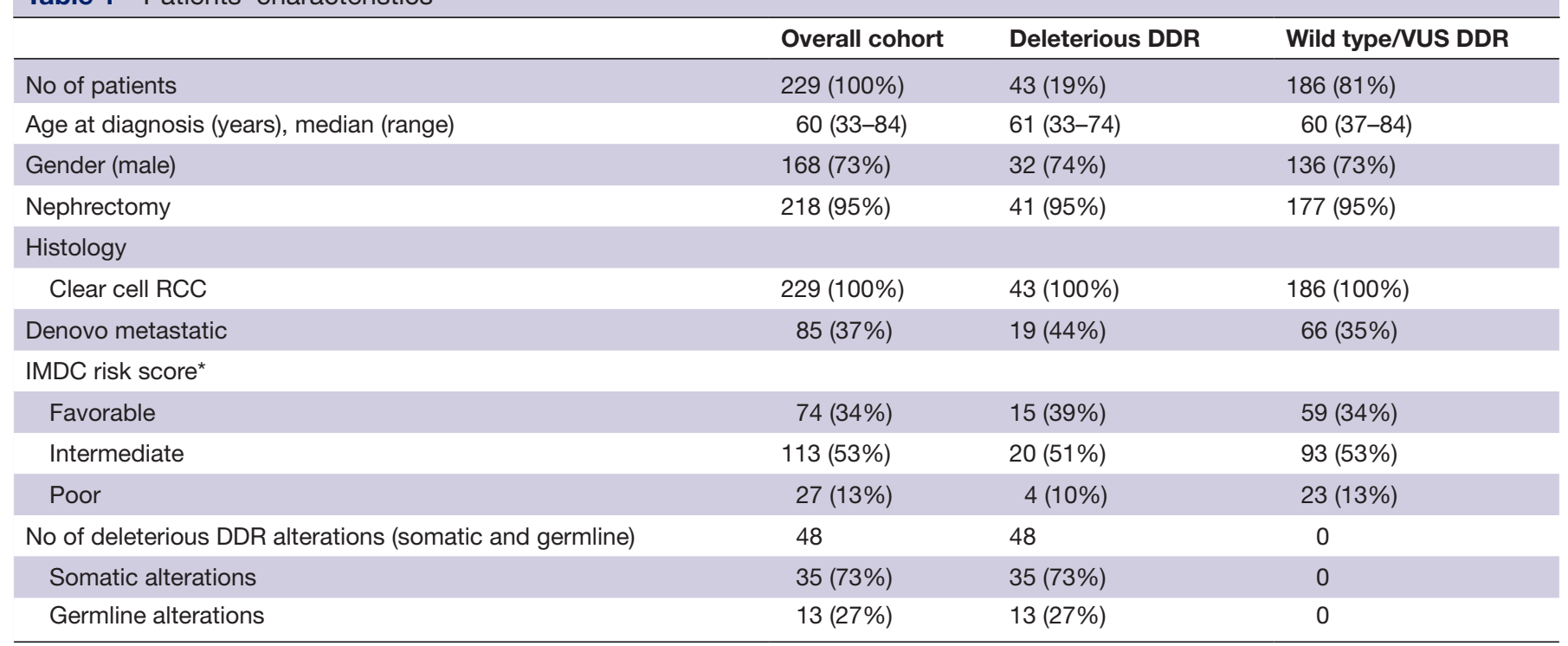

*Missing for 15 patients.

DDR, DNA damage repair; IMDC, International Metastatic Renal Cell Carcinoma Database Consortium; RCC, renal cell carcinoma; VUS, variants of unknown significance. 


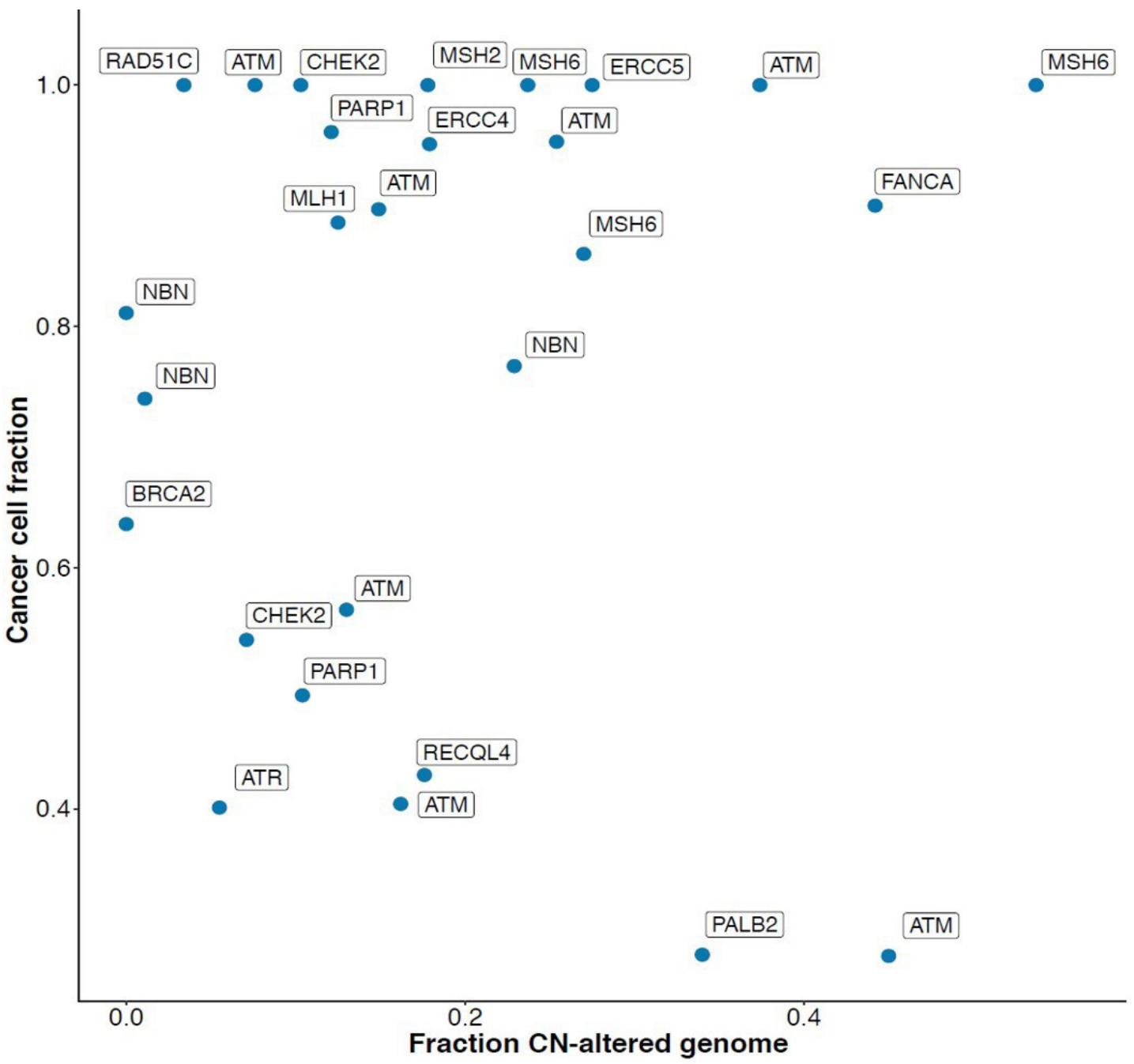

Figure 1 Somatic deleterious DDR gene alterations in metastatic clear cell RCC are commonly clonal. The plot summarizes the distribution of 27 somatic deleterious DDR gene alterations by the CCF (y-axis) and the FCNAg (x-axis). In 17 out of 27 (63\%) deleterious somatic DDR mutations, the CCF probability was $\geq 0.75$ indicative of a higher likelihood that the mutations are clonal. CCF, cancer cell fraction; CN, copy number; DDR, DNA damage repair; FCNAg, fraction of copy number-altered genome; RCC, renal cell carcinoma.

Clinical characteristics of patients harboring deleterious DDR alterations versus those whose tumors were DDR wild type/VUS are summarized in table 1 . CCF analysis to determine clonality was estimable for 27 somatic deleterious DDR gene mutations. The analysis failed in tumors of eight somatic DDR alterations and was not performed for the germline alterations. For the majority of these $(n=17 ; 63 \%)$, the CCF was $\geq 0.75$, indicative of a higher likelihood that the mutations are clonal and represent early events in the disease process (figure 1).

\section{Patients' characteristics in the $\mathrm{I} / 0$ and TKI analysis}

Pertinent clinical features at the time of starting $\mathrm{I} / \mathrm{O}$ and TKI therapies are summarized in tables 2 and 3, respectively. A total of 107 patients contributed to the $\mathrm{I} / \mathrm{O}$ analysis, a significant proportion of these $(63 \%)$ having received prior lines of systemic therapy (see table 2 ). Seventy-three patients $(68 \%)$ received single-agent I/O therapy; the remainder (32\%) received I/O combination therapy, predominantly anti-PD-1+anti-CTLA-4 directed. IMDC risk categories at the start of I/O therapy were favorable/intermediate/poor in $21 \% / 61 \% / 18 \%$, respectively.

A total of 118 patients contributed to the TKI analysis, all having received monotherapy with a VEGF-directed TKI in the first-line setting, most commonly sunitinib (62\%). IMDC risk categories at the start of first-line VEGF-TKI were favorable/intermediate/poor in $29 \% / 58 \% / 13 \%$, respectively. Fifty-four patients contributed data to both the I/O and the TKI analyses.

\section{Association between DDR status and outcomes to systemic therapy}

Survival outcomes in the $1 / 0$ and TKI analyses

In the I/O analysis, there was a total of 47 deaths for 107 patients. Median OS was 50.0 months (95\% CI: 32.6-NE) with a median follow-up time for survivors of 35.8 months (range: 12-85). Median OS was not reached (95\% CI: NE-NE) in the deleterious DDR group versus 39.4 months (95\% CI: 26.7-NE) in the wild-type/VUS DDR group 
Table 2 Characteristics of 107 patients in the $\mathrm{I} / \mathrm{O}$ analysis

\begin{tabular}{|c|c|c|c|}
\hline & All $(n=107)$ & Deleterious DDR $(n=19)$ & Wild type/VUS DDR $(n=88)$ \\
\hline Age at start of treatment (years), median (range) & $61(40-81)$ & $60(40-74)$ & $61(44-81)$ \\
\hline Gender (male) & $78(73 \%)$ & $13(68 \%)$ & $65(74 \%)$ \\
\hline Nephrectomy & $100(93 \%)$ & $18(95 \%)$ & $82(93 \%)$ \\
\hline \multicolumn{4}{|l|}{ IMDC risk score at starting I/O therapy } \\
\hline Favorable & $23(21 \%)$ & $2(11 \%)$ & $21(24 \%)$ \\
\hline Intermediate & $65(61 \%)$ & $15(78 \%)$ & $50(57 \%)$ \\
\hline Poor & $19(18 \%)$ & $2(11 \%)$ & $17(19 \%)$ \\
\hline \multicolumn{4}{|l|}{ I/O therapy type } \\
\hline Anti-PD-1 monotherapy & $61(57 \%)$ & $10(53 \%)$ & $51(58 \%)$ \\
\hline Anti-PD-L1 monotherapy & $12(11 \%)$ & $3(16 \%)$ & $9(10 \%)$ \\
\hline Anti-PD-1+anti-CTLA-4 & $32(30 \%)$ & $5(26 \%)$ & $27(31 \%)$ \\
\hline Anti-PD-1+anti-PD-L1 & $2(2 \%)$ & $1(5 \%)$ & $1(1 \%)$ \\
\hline \multicolumn{4}{|l|}{ I/O therapy category } \\
\hline Monotherapy & $73(68 \%)$ & $13(68 \%)$ & $60(68 \%)$ \\
\hline Combination & $34(32 \%)$ & $6(32 \%)$ & $28(32 \%)$ \\
\hline \multicolumn{4}{|l|}{ Line of I/O therapy } \\
\hline First line & $40(37 \%)$ & $8(42 \%)$ & $32(36 \%)$ \\
\hline$\geq$ Second line & $67(63 \%)$ & $11(58 \%)$ & $56(64 \%)$ \\
\hline
\end{tabular}

CTLA-4, cytotoxic T-lymphocyte-associated protein-4; DDR, DNA damage repair; IMDC, International Metastatic Renal Cell Carcinoma Database Consortium; I/O, immune-oncology; PD-1, programmed cell death-1; PD-L1, programmed cell death-ligand 1; VUS, variants of unknown significance.

(log-rank $\mathrm{p}=0.049$ ) (figure 2A). Survival estimates at 24 months were $78.3 \%$ (95\% CI: $51.9 \%-91.2 \%)$ in the deleterious DDR group versus $62.4 \%$ (95\% CI: $51.1 \%-71.8 \%$ ) in the wild-type/VUS DDR group. Applying a multivariate Cox regression model with adjustment for IMDC risk and the number of previous lines of therapy, the OS HR

Table 3 Characteristics of 118 patients in the TKI analysis

\begin{tabular}{|c|c|c|c|}
\hline & All $(n=118)$ & Deleterious DDR $(n=24)$ & $\begin{array}{l}\text { Wild type/VUS DDR } \\
(n=94)\end{array}$ \\
\hline Age at start of treatment (years), median (range) & $60(34-86)$ & $63(34-74)$ & $60(37-86)$ \\
\hline Gender (male) & $87(74 \%)$ & $20(83 \%)$ & $67(71 \%)$ \\
\hline Nephrectomy & $110(93 \%)$ & $23(96 \%)$ & $87(92 \%)$ \\
\hline \multicolumn{4}{|l|}{ IMDC risk score at starting VEGF-TKI therapy } \\
\hline Favorable & $32(29 \%)$ & $5(25 \%)$ & $27(30 \%)$ \\
\hline Intermediate & $63(58 \%)$ & $11(55 \%)$ & $52(59$ \\
\hline Poor & $14(13 \%)$ & $4(20 \%)$ & $10(11 \%)$ \\
\hline \multicolumn{4}{|l|}{ VEGF-TKI agent } \\
\hline Sunitinib & $73(62 \%)$ & $12(50 \%)$ & $61(65 \%)$ \\
\hline Pazopanib & $42(36 \%)$ & $11(46 \%)$ & $31(33 \%)$ \\
\hline Tivozanib & $1(1 \%)$ & 0 & $1(1 \%)$ \\
\hline Axitinib & $1(1 \%)$ & 0 & $1(1 \%)$ \\
\hline Sorafenib & $1(1 \%)$ & $1(4 \%)$ & 0 \\
\hline \multicolumn{4}{|l|}{ Line of TKI therapy } \\
\hline First line & $118(100 \%)$ & 24 (100\%) & 94 (100\%) \\
\hline
\end{tabular}

DDR, DNA damage repair; IMDC, International Metastatic Renal Cell Carcinoma Database Consortium; TKI, tyrosine kinase inhibitor; VEGF, vascular endothelial growth factor; VUS, variants of unknown significance. 

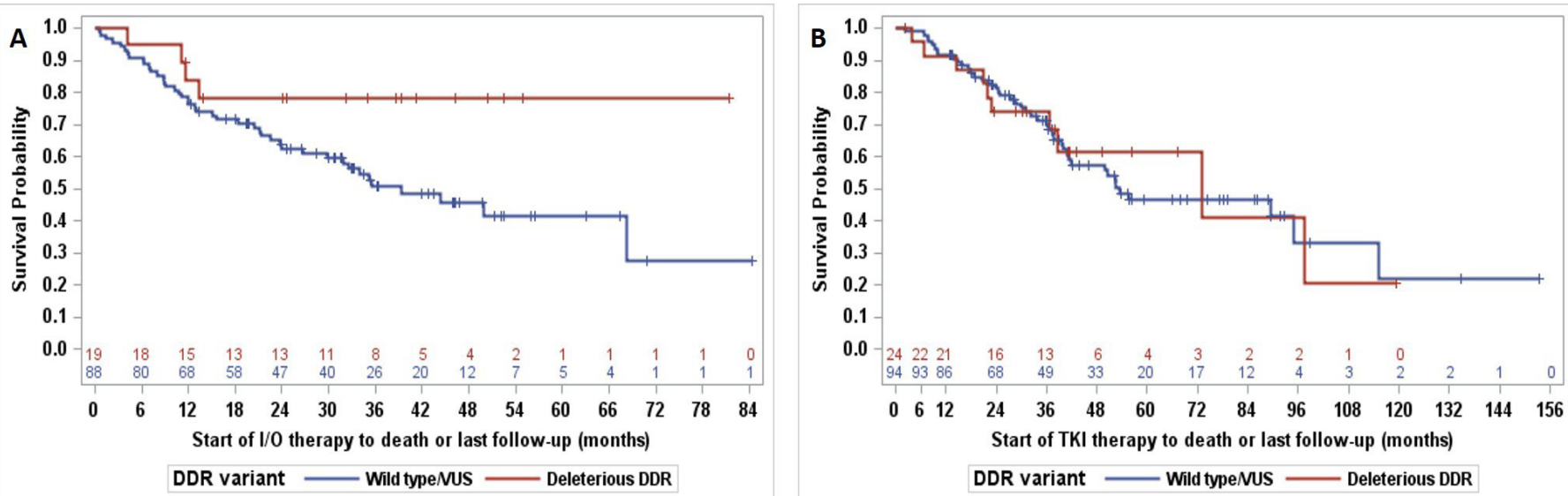

Figure 2 Kaplan-Meier estimate of OS according to DDR gene alterations (deleterious DDR versus wild type/VUS DDR) in (A) I/O-treated patients (log-rank $p=0.049$ ) and (B) VEGF-TKI-treated patients (log-rank $p=0.903$ ). After adjustment for IMDC risk and the number of previous lines of therapy, the OS HR for the presence of deleterious DDR in the I/O analysis was 0.41 (95\% Cl: 0.14-1.14; $p=0.09$ ). DDR, DNA damage repair; IMDC, International Metastatic Renal Cell Carcinoma Database Consortium; I/O, immune-oncology; OS, overall survival; TKI, tyrosine kinase inhibitor; VEGF, vascular endothelial growth factor; VUS, variants of unknown significance.

for the presence of deleterious DDR was 0.41 (95\% CI: $0.14-1.14 ; \mathrm{p}=0.09)$.

In the TKI analysis, there was a total of 53 deaths for 118 patients. Median OS was 55.7 months (95\% CI: 41.1-97.6) with a median follow-up time for survivors of 41 months (range: 2-153). Median OS was 72.9 months (95\% CI: 36.7-NE) in the deleterious DDR group versus 53.6 months (95\% CI: 40.8-115.1) in the wild-type/VUS DDR group (log-rank $\mathrm{p}=0.903$ ) (figure 2B). Survival estimates at 24 months were $73.9 \%$ (95\% CI: $50.9 \%-87.3 \%$ ) in the deleterious DDR group versus $81.3 \%$ (95\% CI: $71.6 \%-$ $88.0 \%$ ) in the wild-type/VUS DDR group. In this analysis of patients uniformly treated in the first-line setting, Cox regression with adjustment for IMDC risk determined the HR for the presence of deleterious DDR to be $1.06(95 \%$ CI: $0.49-2.29$; $\mathrm{p}=0.88$ ).

In the I/O analysis, median PFS for 94 patients with serial radiographic assessments per RECIST V.1.1 was
5.3 months (95\% CI: 4.0-8.1), with a total of 66 disease progression events. Median PFS was comparable for patients harboring deleterious DDR alterations and those whose tumors were DDR wild type/VUS (4.0 months; 95\% CI: 2.7-NE; and 5.3 months; 95\% CI: 4.1-9.8; respectively; log-rank $\mathrm{p}=0.930$ ) (figure $3 \mathrm{~A}$ ). Similarly, no differences were seen in 85 patients with RECIST V.1.1 assessments on TKI therapy with median PFS across all patients being 10.9 months (95\% CI: 8.7-13.8) and not significantly different for those in the deleterious DDR group (17.1 months; 95\% CI: 8.1-28.1) versus the DDR wild-type/VUS group (9.6 months; 95\% CI: 7.9-12.4; with $\log$-rank $\mathrm{p}=0.329$ ) (figure $3 \mathrm{~B}$ ). The total progression disease events in the TKI analysis were 70. Swimmers plots in online supplementary figures S1 and S2 visualize the time course of therapy for 19 and 24 patients in the deleterious DDR group in the I/O and TKI analyses, respectively.
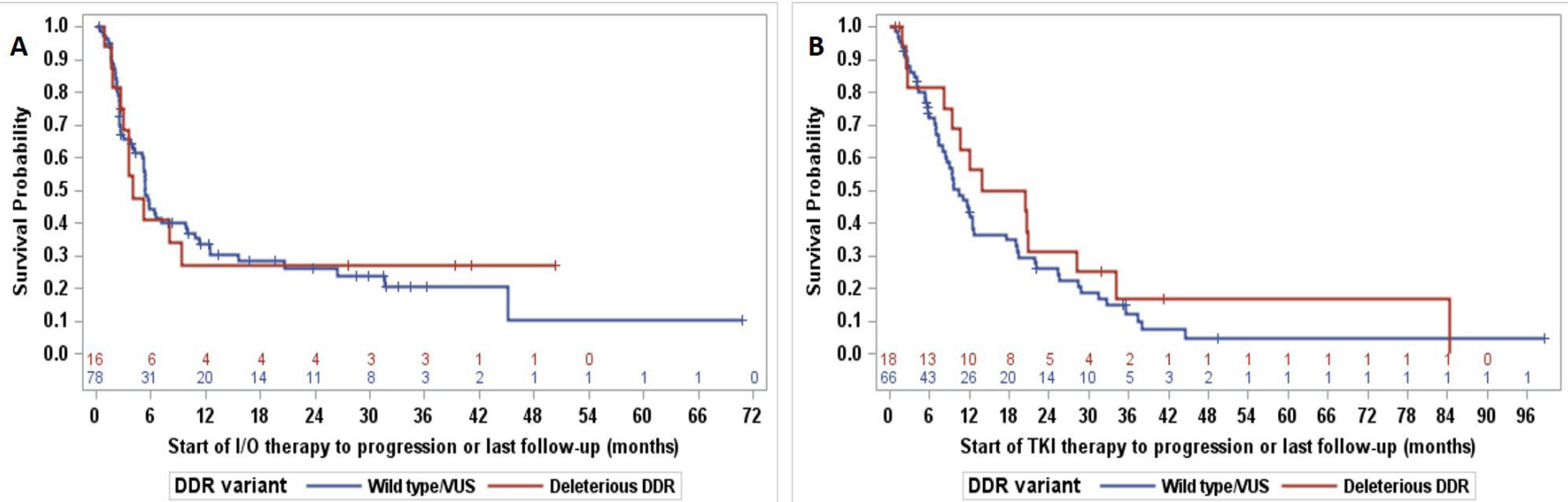

Figure 3 Kaplan-Meier estimate of PFS according to DDR gene alterations (deleterious DDR vs wild type/VUS DDR) in (A) I/O-treated patients (log-rank $p=0.930$ ) and (B) VEGF-TKI-treated patients (log-rank $p=0.329)$. DDR, DNA damage repair; l/O, immune-oncology; PFS, progression-free survival; TKI, tyrosine kinase inhibitor; VEGF, vascular endothelial growth factor; VUS, variants of unknown significance. 
We further conducted a sensitivity OS analysis in patients who only underwent NGS in the primary tumor samples in both the I/O and the TKI analyses (64 and 75 patients, respectively); we observed a similar trend toward better OS in the I/O-treated patients and not in the TKItreated patients (HR 0.42 (95\% CI: $0.13-1.37$ ); $\mathrm{p}=0.13$ vs HR 0.94 (95\% CI: 0.43-2.07); $\mathrm{p}=0.88$ ); however, this was not statistically significant probably underpowered by the small number of patients in each analysis (online supplementary figure S3).

\section{Radiographic responses in the $\mathrm{l} / 0$ and TKI analyses}

For 94 patients with RECIST V.1.1 evaluation in the I/O analysis, the ORR was $32 \%$. There was no significant difference in ORR between the deleterious DDR group and wild-type/VUS DDR group (38\% vs $31 \%$; Fisher's exact $\mathrm{p}=0.57$ ) (online supplementary table S3). In the $\mathrm{I} / \mathrm{O}$ analysis, four of the nine patients with germline DDR events (44\%) achieved an objective response. For 85 patients with RECIST V.1.1 evaluation contributing to the TKI analysis, the best ORR was $46 \%$ with no significant difference observed between the deleterious DDR group versus wild-type/VUS DDR group (33\% vs 46\%; Fisher's exact $\mathrm{p}=0.42$ ) (online supplementary table $\mathrm{S} 4$ ). In the TKI analysis, three of the eight patients with germline DDR events $(37 \%)$ achieved an objective response. Co-mutation plots in online supplementary figure S4A,B illustrate the distribution of the deleterious DDR gene alterations by the relevant genes and pathways with the radiographic responses for each patient in both the I/O and the TKI analyses. Waterfall plots in online supplementary figure S5A,B depict the maximal amounts of shrinkage in target lesions for patients highlighting DDR mutation status in the I/O and TKI analyses, respectively.

\section{DISCUSSION}

Damage to human DNA occurs constantly as a result of exposure to a multitude of endogenous and exogenous influences. The DDR machinery reacts to such events through a number of well-orchestrated mechanisms that can regulate DNA replication and may lead to the repair or bypass of DNA damage as well as cell cycle arrest. ${ }^{82}$ These response pathways are regulated by several key DDR genes, functional loss of which can result in genomic instability and frequently contributes to neoplastic transformation. DDR status is understood to have implications on neoantigen load and hence has been explored as a potential predictor of clinical benefit to I/O agents. The activity of $\mathrm{I} / \mathrm{O}$ in this setting was first highlighted in the pivotal phase II study of pembrolizumab, ${ }^{11}$ which was conducted primarily in colon cancer and demonstrated that patients with deficient mismatch repair (dMMR) tumors had higher response rates compared with MMRproficient tumors (40\% vs $0 \%$ ). These findings were subsequently confirmed in a larger phase II study ${ }^{23}$ evaluating pembrolizumab solely in patients with dMMR tumors across 12 tumors types (none of which were RCC) with an ORR of 53\%, including 21\% complete responses. This discovery led to the Food and Drug Administration approval of pembrolizumab for the treatment of MSI-H/ dMMR cancers, irrespective of primary tumor site. ${ }^{24}$ In a previously reported study of 60 patients with metastatic urothelial cancers enrolled in prospective trials who were treated with a variety of $\mathrm{I} / \mathrm{O}$-based regimens, ${ }^{12}$ the presence of deleterious DDR gene alterations across the same set of candidate genes tested here was associated with superior OS. Similarly, Wang et $a l^{25}$ recently demonstrated that the presence of concomitant mutations in HRR-MMR or HRR-BER pathways was associated with clinical benefit to $\mathrm{I} / \mathrm{O}$ agents across several cancer types.

In pan-cancer comparisons, a genotype suggestive of genomic instability is more common in RCC than other solid tumor malignancies, ${ }^{26}{ }^{27}$ yet dedicated reports around DDR biology in this disease are limited. In the localized setting, data from The Cancer Genome Atlas (TCGA) effort have provided insights into genomics and transcriptomics of DNA maintenance in ccRCC. ${ }^{27} 28$ Here, alterations across 11 DDR genes were found in 25\% of cases. In a subsequent report from the Kidney Renal Clear Cell Carcinoma (KIRC) TCGA dataset, transcriptomic signatures suggestive of HR deficiency were seen in the majority of cases. ${ }^{29}$

Our study extends the exploration of DDR genomics to the metastatic setting, analyzing a large retrospective cohort of systemically treated patients. To our knowledge, this is the first report to put such findings into context with different categories of systemic RCC therapy. In our cohort of $>200$ patients, we confirm that loss-of-function events in key DDR genes are common in the setting of advanced disease and report deleterious DDR gene alterations in $19 \%$ of patients, the majority deemed clonal per CCF estimates.

Following prior reports in other malignancies, we were interested to explore the significance of DDR loss-offunction and therapeutic outcomes in RCC. We undertook separate analyses for I/O and VEGF-TKI agents. Importantly, this retrospective study did not allow for direct comparison between treatment groups, and we did not attempt to undertake such comparison. While there were no differences for PFS or ORR, longer median OS with $\mathrm{I} / \mathrm{O}$ therapy was observed in patients whose tumors harbored deleterious DDR gene alterations versus those who were DDR WT/VUS (log-rank $\mathrm{p}=0.049$ ). Landmark analysis with 24 months OS probability also favored DDRaltered cases. Significance of this OS association was lost in a multivariate Cox regression model correcting for IMDC risk category and exposure to prior lines of therapy (HR 0.41, 95\% CI: $0.14-1.14 ; \mathrm{p}=0.09$ ), which may be due to interdependence or due to limitations in sample size. The provocative signal seen here for OS with a lack of concurrent association in ORR and PFS highlights the complexities of interpreting $\mathrm{I} / \mathrm{O}$ benefit previously evident in other RCC datasets. ${ }^{60}$ Small sample sizes of the various subgroups did not allow for pathway or gene-specific exploration of an efficacy signal. We did not appreciate 
association between DDR status and median PFS, median OS, 24 months OS or ORR in patients treated with firstline TKI therapy $(\log$-rank $\mathrm{p}=0.903)$.

In 10 patients, deleterious DDR status was determined solely by the presence of germline DDR events, mostly involving CHEK2 and MUTYH. When looking at this small number of patients, no notable differences in treatment effect, including survival, were apparent between the somatic and germline variants.

Our results add to the growing level of evidence supporting the notion that DDR status deserves further study in the context of treating metastatic ccRCC with $\mathrm{I} / \mathrm{O}$ agents. For ccRCC, validation in larger, prospective cohorts is needed, ideally with dedicated attention to particular pathways and individual genes. Better understanding of these concepts may also allow for rational combination strategies pairing $\mathrm{I} / \mathrm{O}$ and targeted agents. This includes Poly (ADP-ribose) polymerase (PARP) inhibitors for which synthetic lethality in a DDR-impaired state has been proposed in preclinical RCC models. ${ }^{31}$

Our study has several limitations. All patients in this retrospective cohort were treated at a single center, and as mentioned sample size limits our ability to correct for other factors and explores the significance of individual genes. A proportion of patients contributed to both TKI and $\mathrm{I} / \mathrm{O}$ analyses, although it should be stressed that the purpose of our study was not to compare outcomes between different therapeutic approaches. The clinical setting was rather different, TKI therapy having been applied in the first line for all patients analyzed here while a significant proportion of $\mathrm{I} / \mathrm{O}$ treatments was initiated after prior exposure to non-I/O therapies. Relevantly, no patients in this study entered either analysis on the basis of I/O plus TKI combination therapy. OS data seen in this cohort exceed historic controls for first-line TKI treatments. ${ }^{3}$ Such differences are partly attributable to the bias inherent to retrospective research but may also reflect what can be achieved in patients with early access to a contemporary standard and investigational agents at a center of expertise. Additionally, genomic characterization of DDR pathways and estimation of mutational load were limited to genes included in the MSK-IMPACT gene panel; this is a targeted NGS panel containing a limited number of genes (468 in the most recent version), which represents a fraction of the genome when compared with other extensive assays such as compared with whole exome sequencing.

\section{CONCLUSIONS}

Our findings add further evidence that DDR alterations are frequent loss-of-function events in metastatic ccRCC and support the notion that this category of genomic alterations deserves further study investigating their predictive value for $\mathrm{I} / \mathrm{O}$ agents in this disease and others. This was an exploratory study to gain early insight into the significance of DDR gene function in metastatic RCC with respect to treatment with standard agents. With a retrospective design and limited sample size, it was not set up to establish an applicable biomarker for I/O therapy in RCC.

\section{Author affiliations}

${ }^{1}$ Department of Medicine, Memorial Sloan Kettering Cancer Center, New York, New York, USA

${ }^{2}$ Department of Radiology, Memorial Sloan Kettering Cancer Center, New York, New York, USA

${ }^{3}$ Department of Surgery, Memorial Sloan Kettering Cancer Center, New York, New York, USA

${ }^{4}$ Department of Epidemiology and Biostatistics, Memorial Sloan Kettering Cancer Center, New York, New York, USA

${ }^{5}$ Department of Radiation Oncology, Memorial Sloan Kettering Cancer Center, New York, New York, USA

Contributors Concept and design: YG, RGD, C-HL, MYT, RJM, MHV. Administrative support: AF. Collection and assembly of data: YG, JLC, RGD, AF. Data analysis and interpretation: YG, RGD, AK, RRK, MIC, C-HL, DRF, MYT, SP, RJM, AAH, MHV. Manuscript writing: all authors. Final approval of manuscript: all authors.

Funding Precision Immunotherapy Kidney Cancer Program, Center Core Grant P30 CA008748 from the National Cancer Institute.

Competing interests MIC reports consulting/advisory role for Pfizer. C-HL reports consulting/advisory role for Exelixis and Eisai. DRF reports research support from Novartis and Seattle Genetics. TAC reports research support from Bristol-Myers Squibb, AstraZeneca, Eisai, An2H and Illumina. TAC holds a patent for the use of TMB to predict immunotherapy response. This is licensed to PGDx and MSKCC and TAC are entitled to receive royalties. TAC is a cofounder of Gritstone Oncology and holds equity. RJM reports grants and personal fees from Pfizer, grants and personal fees from Eisai, personal fees from Exelixis, grants and personal fees from Novartis, grants from Bristol-Myers Squibb, grants and personal fees from Genentech/Roche, personal fees from Merck, personal fees from Incyte, grants from GlaxoSmithKline, outside the submitted work. MHV reports receiving commercial research grants from Bristol-Myers Squibb and Genentech/Roche. Honoraria from Novartis. Travel/accommodation from Eisai, Novartis and Takeda. Consultant/advisory board member for Alexion Pharmaceuticals, Bayer, Calithera Biosciences, Corvus Pharmaceuticals, Exelixis, Eisai, GlaxoSmithKline, Natera, Novartis and Pfizer.

Patient consent for publication Not required.

Ethics approval Retrospective chart review was conducted under Memorial Sloan Kettering Cancer Center (MSKCC) IRB protocol \#18-451. Next generation sequencing data were collected using MSKCC-IRB protocol \#12-245 for which the participants provided a signed informed consent.

Provenance and peer review Not commissioned; externally peer reviewed. Data availability statement Data are available upon reasonable request.

Open access This is an open access article distributed in accordance with the Creative Commons Attribution Non Commercial (CC BY-NC 4.0) license, which permits others to distribute, remix, adapt, build upon this work non-commercially, and license their derivative works on different terms, provided the original work is properly cited, appropriate credit is given, any changes made indicated, and the use is non-commercial. See http://creativecommons.org/licenses/by-nc/4.0/.

\section{ORCID iDs}

Ritesh R Kotecha http://orcid.org/0000-0002-0223-3479

Robert J Motzer http://orcid.org/0000-0001-6925-2327

Martin H Voss http://orcid.org/0000-0003-0551-5807

\section{REFERENCES}

1 Choueiri TK, Motzer RJ. Systemic therapy for metastatic renal-cell carcinoma. N Engl J Med 2017;376:354-66.

2 Motzer RJ, Hutson TE, Tomczak P, et al. Sunitinib versus interferon alfa in metastatic renal-cell carcinoma. $N$ Engl J Med 2007;356:115-24.

3 Motzer RJ, Hutson TE, Cella D, et al. Pazopanib versus sunitinib in metastatic renal-cell carcinoma. N Engl J Med 2013;369:722-31.

4 Rini BI, Escudier B, Tomczak P, et al. Comparative effectiveness of axitinib versus sorafenib in advanced renal cell carcinoma (axis): a randomised phase 3 trial. Lancet 2011;378:1931-9. 
5 Choueiri TK, Escudier B, Powles T, et al. Cabozantinib versus everolimus in advanced renal-cell carcinoma. N Engl J Med 2015;373:1814-23.

6 Motzer RJ, Escudier B, McDermott DF, et al. Nivolumab versus everolimus in advanced renal-cell carcinoma. $N$ Engl J Med 2015;373:1803-13.

7 Motzer RJ, Tannir NM, McDermott DF, et al. Nivolumab plus ipilimumab versus sunitinib in advanced renal-cell carcinoma. N Engl J Med 2018;378:1277-90.

8 Harper JW, Elledge SJ. The DNA damage response: ten years after. Mol Cell 2007;28:739-45.

9 Sen T, Rodriguez BL, Chen L, et al. Targeting DNA damage response promotes antitumor immunity through STING-mediated T-cell activation in small cell lung cancer. Cancer Discov 2019;9:646-61.

10 Barber GN. Sting: infection, inflammation and cancer. Nat Rev Immunol 2015;15:760-70.

11 Le DT, Uram JN, Wang H, et al. PD-1 blockade in tumors with mismatch-repair deficiency. N Engl J Med 2015;372:2509-20.

12 Teo MY, Seier K, Ostrovnaya I, et al. Alterations in DNA damage response and repair genes as potential marker of clinical benefit from PD-1/PD-L1 blockade in advanced urothelial cancers. J Clin Oncol 2018;36:1685-94.

13 Cheng DT, Mitchell TN, Zehir A, et al. Memorial Sloan KetteringIntegrated mutation profiling of actionable cancer targets (MSKIMPACT): a hybridization capture-based next-generation sequencing clinical assay for solid tumor molecular oncology. J Mol Diagn 2015;17:251-64.

14 Vivian J, Rao AA, Nothaft FA, et al. Toil enables reproducible, open source, big biomedical data analyses. Nat Biotechnol 2017;35:314-6.

15 Shen R, Seshan VE. FACETS: allele-specific copy number and clonal heterogeneity analysis tool for high-throughput DNA sequencing. Nucleic Acids Res 2016;44:e131.

16 Endesfelder D, Burrell R, Kanu N, et al. Chromosomal instability selects gene copy-number variants encoding core regulators of proliferation in ER+ breast cancer. Cancer Res 2014;74:4853-63.

17 Tate JG, Bamford S, Jubb HC, et al. COSMIC: the Catalogue of somatic mutations in cancer. Nucleic Acids Res 2019;47:D941-7.

18 Shihab HA, Rogers MF, Gough J, et al. An integrative approach to predicting the functional effects of non-coding and coding sequence variation. Bioinformatics 2015;31:1536-43.

19 Richards S, Aziz N, Bale S, et al. Standards and guidelines for the interpretation of sequence variants: a joint consensus recommendation of the American College of medical genetics and genomics and the association for molecular pathology. Genet Med 2015;17:405-23.

20 McGranahan N, Favero F, de Bruin EC, et al. Clonal status of actionable driver events and the timing of mutational processes in cancer evolution. Sci Transl Med 2015;7:283ra54.

21 Eisenhauer EA, Therasse P, Bogaerts J, et al. New response evaluation criteria in solid tumours: revised RECIST guideline (version 1.1). Eur J Cancer 2009;45:228-47.

22 Hoeijmakers JH. Genome maintenance mechanisms for preventing cancer. Nature 2001;411:366-74.

23 Le DT, Durham JN, Smith KN, et al. Mismatch repair deficiency predicts response of solid tumors to PD-1 blockade. Science 2017;357:409-13.

24 U.S. Food and Drug Administration. FDA grants accelerated approval to pembrolizumab for first tissue/site agnostic indication, 2017. Available: https://www.fda.gov/Drugs/InformationOnDrugs/ ApprovedDrugs/ucm560040.htm

25 Wang Z, Zhao J, Wang G, et al. Comutations in DNA damage response pathways serve as potential biomarkers for immune checkpoint blockade. Cancer Res 2018;78:6486-96.

26 Alexandrov LB, Nik-Zainal S, Wedge DC, et al. Signatures of mutational processes in human cancer. Nature 2013;500:415-21.

27 Cancer Genome Atlas Research Network. Comprehensive molecular characterization of clear cell renal cell carcinoma. Nature 2013;499:43-9.

$28 \mathrm{Na}$ JC, Nagaya N, Rha KH, et al. DNA damage response pathway alteration in locally advanced clear-cell renal-cell carcinoma is associated with a poor outcome. Clin Genitourin Cancer 2019;17:299-305

29 Pilie PG, Peterson CB, Peng Y, et al. Functional biomarkers of homologous repair (HR) deficiency to guide novel DNA damage response targeted therapy in clear cell renal cell carcinoma (ccRCC). Journal of Clinical Oncology 2018;36:4576.

30 Motzer RJ, Rini BI, McDermott DF, et al. Nivolumab for metastatic renal cell carcinoma: results of a randomized phase II trial. J Clin Oncol 2015;33:1430-7.

31 Scanlon SE, Hegan DC, Sulkowski PL, et al. Suppression of homology-dependent DNA double-strand break repair induces PARP inhibitor sensitivity in $\mathrm{VHL}$-deficient human renal cell carcinoma. Oncotarget 2018:9:4647-60. 